\title{
Early lethality of embryos derived from transgenic Xenopus females is associated with reduced ovarian grem 1 expression
}

\author{
Beck $\mathrm{CW}^{1 *}$, Ward $\mathrm{J}^{1}$, Troise $\mathrm{L}^{1,2}$ and Brochard $\mathrm{C}^{1,2}$ \\ ${ }^{1}$ Department of Zoology, University of Otago, Dunedin, New Zealand \\ ${ }^{2}$ Current Address: Ecole Nationale Vétérinaire de Toulouse, Toulouse, France
}

\begin{abstract}
The grem1 gene codes a protein that inhibits the action of multiple members of a growth factor family known as bone morphogenetic proteins (BMPs). Certain members of this BMP family can regulate both fecundity and fertility in mammals via their action on oocyte (egg) development, and Grem1 has been identified as a marker of oocyte quality in humans. The model amphibian Xenopus laevis is far more fecund than mammals, producing thousands of eggs in a clutch. Female transgenic frogs carrying grem 1 under the control of a stress inducible $b s p 70$ promoter ("G" frogs) produce very few viable offspring. Here, we show that this is not due to reduced fecundity or fertilization rate, but results from a significant reduction in subsequent survival over the first day of development. Embryos that successfully survive for the first day were found to go on to develop normally when compared to their peers. Both the morphology and stage distribution of oocytes from $\mathrm{G}$ females appears normal, and oocytes develop at expected rates, although stage VI oocytes were found to have a lower response to in vitro progesterone treatment. Unexpectedly, levels of grem1 mRNA were found to be consistently lower in the female ovaries from four independent $\mathrm{G}$ transgenic lines than in wild type ovaries, indicating that the reduced early survival rate of offspring was not due to placement of, or leaky expression from, the transgene. Both transgenic and wild type offspring were equally affected, confirming a maternal effect. Our study shows that transgenic females with the lowest levels of grem 1 transcripts in the ovary have the lowest rates of survival past the first day of amphibian embryogenesis, equivalent to pre-implantation staged mammalian embryos. The reduced expression of grem 1 in the oocytes of transgenic females suggests transgene suppression of an endogenous locus may occur in the Xenopus female germline, an unexpected finding.
\end{abstract}

\section{Introduction}

Infertility, which affects 70 million human couples worldwide, can have a genetic basis [1]. Female infertility can result from defective development of the oocyte, and two of the very few genes associated with this kind of infertility belong to the same family of growth factors: the bone morphogenetic proteins (BMPs). In humans, both BMP15 $[2,3]$ and its close relative GDF9 (growth and differentiation factor 9) [4] are implicated in fertility. Both GDF9 and BMP15 are actively transcribed in developing oocytes, although not exclusively. In ewes, heterozygous mutations in GDF9 or BMP15 can increase fecundity by increasing the rate of ovulation, but homozygous mutations result in infertility [5-7]. BMP15 and GDF9 are therefore targets for fertility regulation in sheep and cattle [8]. However, in mice, which have larger litters, while GDF9 loss results in infertility [9] loss of BMP15 only reduces litter size [10]. As well as GDF9 and BMP15 in the oocytes, $B M P 4$ and $B M P 7$ are expressed in the surrounding stroma during oogenesis and positively regulate oocyte development. BMPs are secreted proteins that bind as dimers to type I and II receptor serine/ threonine kinases in the plasma membrane of target cells and elicit cell signalling via activation of a group of cytoplasmic proteins known as SMADs [11]. Together these form transcriptional complexes, which regulate specific gene expression at BMP response elements [12].

Xenopus laevis, the south African clawed frog, is a useful model for the study of development as eggs can be obtained all year round following injection with human chorionic gonadotrophin (hCG), and these are large enough to be easily manipulated, with a diameter of 1.2 $\mathrm{mm}$. Oogenesis, the progression from primordial germ cell to mature egg, is an asynchronous process in Xenopus, with all stages being present in the ovary throughout adult life. Oogenesis results in the formation of a polarised egg in Xenopus and six stages of oocyte development (I-VI) were identified and described by Dumont [13]. The germ cells (oogonia) first undergo a series of mitotic cell divisions leading to the formation of the primary oocyte. Meiosis then begins, but is arrested in prophase I. The oocyte then undergoes a period of growth, and uptake of nutrients, such as vitellogenin: Dumont stages II to V are considered vitellogenic, stage VI is post-vitellogenic. Animal-vegetal polarity is established as pigment is displaced to one pole and mitochondria to the other. In the response to hCG, progesterone is produced from ovarian follicle cells and induces the resumption of meiosis in stage VI oocytes. The germinal vesicle (nucleus) migrates to the animal pole where it is visible as a white spot, forming a visible marker of the process, and the first polar body is lost. The resulting mature oocyte (egg) is arrested at metaphase II pending fertilization. Once this occurs, the egg completes meiosis and the second polar body is lost.

BMPs are potent morphogens, able to elicit distinct cellular responses at different concentrations, and so their dispersal is regulated by a group of extracellular inhibitors [14]. Grem1 encodes Gremlin1, a cysteine knot protein that acts as an extracellular inhibitor of several members of the bone morphogenetic protein (BMP) family [15]. Gremlin1 binds directly to BMPs preventing them activating their receptors. In our laboratory, we have generated several lines of transgenic Xenopus laevis in order to study the role of Gremlin1 in limb

${ }^{\star}$ Correspondence to: Caroline Beck, Department of Zoology, University of Otago, Dunedin, New Zealand, E-mail: caroline.beck@otago.ac.nz

Received: August 20, 2018; Accepted: August 28, 2018; Published: August 30, 2018 
development and regeneration [16]. These animals carry the double transgene hsp70:grem1, $\gamma$-crystallin:RFP (Figure 1a). Animals carrying this transgene are known as $\mathrm{G}$, and each founder has a unique number (Figure 1b,c). These animals can be "genotyped" by the presence of red fluorescent protein (RFP) in the lens of the eye. While they do not express grem 1 from the transgene heat shock protein 70 ( $h s p 70)$ promoter at ambient temperatures, following a heat shock (transfer of animals to $34^{\circ} \mathrm{C}$ for 30 minutes), they rapidly express grem 1 in all cells [16]. However, even at ambient temperatures, female frogs carrying the $G$ transgene were unexpectedly observed to exhibit reduced fertility, so these previous studies relied on the use of male carriers. Intriguingly, grem 1 had already been associated with fertility in humans. Increased grem1 transcription in human cumulus granulosa cells (GC) is associated with better blastocyst formation, embryo quality and improved pregnancy success rate $[17,18]$. The aim of the study was to find the underlying cause of reduced viable offspring from $G$ mothers and to determine how the transgene insertion could cause this.

\section{Materials and methods}

\section{Ethics approval}

All protocols involving animals used in this study were approved by the University of Otago's Animal ethics committee under protocol AEC\#97/15.

\section{Transgenic animals and crosses}

$G$ transgenic founder frogs contain a random insertion of the hsp70:grem1, $\gamma$-crystallin:RFP (G) transgene (Figure 1a). Transgenic animals used in this study were generated using sperm nuclear injection for a previous project, and their design is described therein [16]. Adult females were between 2 and 6 years old at the time of the study. Adult males were between 1 and 6 years old. As founders are produced by titrated random transgene insertion into fertilized eggs, these animals are expected to be heterozygous for the transgene. Five male founders (G5, G6, G7, G8 and G14) were crossed to a single wildtype (WT) female as well as to individual WT females to determine paternal effects (Figure 1b). Three female founders (G3, G9 and G12) and four F1 females (G8.x) descended from male founder G8 were used to determine maternal effect and transgene position effect, by crossing to the same WT male (Figure 1c).

\section{Fecundity assay}

Adult female Xenopus laevis were first weighed and then induced to ovulate by injecting $6.7 \mathrm{U}$ hCG/g bodyweight (i.e. $500 \mathrm{U}$ for a $75 \mathrm{~g}$ female) into the dorsal lymph sac. Injected females were housed in pairs in an incubator at $17^{\circ} \mathrm{C}$ for 16 hours overnight. In the morning, females were individually transferred to $1 \mathrm{x}$ MMR (Marc's modified ringers). Once egg laying had commenced, the number of eggs laid in one hour was counted for each individual. Due to the strong positive correlation between WT female bodyweight before induction and the number of eggs laid in one hour (Figure 2a), the data were normalized for each animal by dividing raw counts by bodyweight in grams and multiplying by $62 \mathrm{~g}$, the median frog weight in this study.

\section{In vitro fertilization rate}

All in vitro fertilization was done using naturally laid eggs, with individual females housed in 1x MMR as above, to prevent activation. Sperm suspension was prepared by gently homogenising the testes from a euthanized male frog in $1 \mathrm{ml} 1 \mathrm{x}$ MMR using a plastic pestle. Approximately 100 eggs were transferred to $50 \mathrm{~mm}$ petri dishes with minimal $1 \times$ MMR and fertilized by adding $20 \mu \mathrm{l}$ sperm suspension, waiting 1-2 minutes, and then flooding eggs with water. Three replicates of each fertilization were performed. Eggs were scored as fertilized once the embryo had begun to cleave, and fertility was defined as the percentage of eggs reaching the 2-4 cell stage.
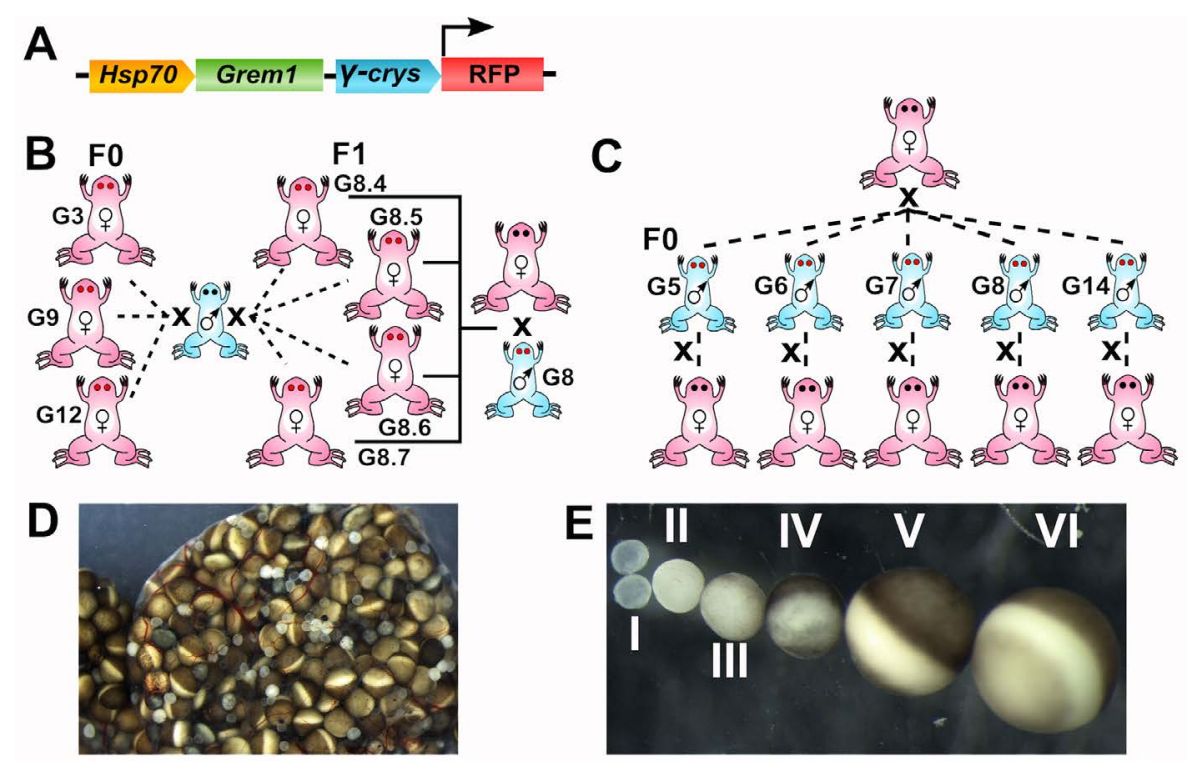

Figure 1. Design of transgene and parent of origin crossing scheme and oocyte staging

A) Schematic of $G$ transgene as published and characterised in [16]. At the temperatures used to house frogs and raise embryos, the $h s p 70$ promotor is expected to be silent [16], so no transgene derived grem 1 transcripts are detectable. The $\gamma$-crystallin promotor drives expression of red fluorescent protein in the lenses of $G$ tadpoles and frogs, allowing easy genotyping. B) Back crossing scheme used for transgenic female frogs. Founders G3, G9 and G12 as well as 4 female descendants of male founder G8 were crossed to the same wild type (WT) male frog. C) Back crossing scheme used for transgenic male frogs. Founders G5, G6, G7, G8 and G14 were crossed both to the same WT female and also to individual WT females. All founders in this study were heterozygous for the $G$ transgene, but have random insertion sites. D) an ovarian lobe from an uninduced female wild type Xenopus. Note all stages ae present at once. E) Collagenase treated oocytes staged from I to VI as in [13]. 


\section{Determination of rate of survival to 24 hours}

Following fertilization confirmation, embryos were treated with $2 \%$ Cysteine $\mathrm{HCl} \mathrm{pH} 7.9$ in $0.1 \times$ MMR to completely remove the jelly coats, rinsed $3 \times$ with $0.1 \times$ MMR, and the unfertilized eggs disregarded. Remaining embryos were incubated at $18^{\circ} \mathrm{C}$ overnight. 28 hours after initial fertilization, which equates to completion of gastrulation/start of neurulation at this temperature, or Nieuwkoop and Faber stage 14 [19], the number of embryos developing normally in each dish were counted. Counts were also obtained for embryos that had died or undergone abnormal gastrulation, before discarding these. Percentages of normal, abnormal and dead embryos were calculated relative to the number of successfully fertilized eggs, with three replicates for each cross.

\section{Determination of transgenic ratios and survival to six days}

The number of transgenic vs. non-transgenic survivors was determined at day 6 after fertilization, by observing presence or absence of the reporter red fluorescent protein (RFP) in the lenses of the embryonic eyes. As all crosses were heterozygote $\mathrm{x}$ wild type (i.e. back-cross) regardless of the sex of the transgenic animal, half the offspring are expected to carry the transgene. Chi-squared tests were used to determine any variation from the expected Mendelian ratio of RFP to non-RFP animals (50:50). Percentages of surviving, transgenic and non-transgenic embryos were calculated relative to the number of successfully fertilized eggs with three replicates for each cross.

\section{Long-term survival analysis}

Embryos developing normally at 6 days post fertilization were first sorted by transgenic status and the three replicate samples pooled before transferring to tanks to a recirculating aquarium. Daily feeding with spirulina and ground salmon pellets commenced at this stage. The aquarium was kept at $23^{\circ} \mathrm{C}$. Development to 10 weeks post fertilization was assessed after terminal anaesthesia (MS222 overdose) by recording the developmental stage of each individual tadpole using the staging system of Nieuwkoop and Faber [19,20].

\section{Ovary composition and oocyte counting}

Female Xenopus were sacrificed and their ovaries examined after 6 weeks of recovery following hCG induction. A single lobe from each ovary was isolated for each female and placed in a sealed $15 \mathrm{ml}$ tube with $10 \mathrm{ml} 0.2 \%$ collagenase in $1 \mathrm{x}$ MMR to separate the oocytes. Collagenase treatment was performed in a $30^{\circ} \mathrm{C}$ incubator with constant slow endover-end rotation. After 30 minutes, oocytes could be separated from each other and counted by stage according to Dumont [13].

\section{Oocyte maturation assay}

For each female in the study, a total of 20 Stage VI oocytes that had been isolated from collagenase treated ovaries (10 from the left and 10 from the right) were exposed to $1 \mathrm{x}$ MMR containing either $30 \mathrm{uM}$ progesterone (prepared as $30 \mathrm{mM}$ stock in ethanol and used fresh) or vehicle for 10 minutes. The oocytes were then gently rinsed in $1 \mathrm{x}$ MMR and transferred to agar lined petri dishes containing $1 \mathrm{x}$ MMR containing penicillin/streptomycin for 4 hours to overnight. The breakdown of the germinal vesicle (female nucleus) leads to a visible white spot appearing at the animal pole and indicates successful maturation of the oocyte. Oocytes were scored as mature, immature or dead.

\section{Quantitative reverse transcription PCR (qRT-PCR)}

Whole ovary tissue was homogenised in TRIzol and total RNA isolated. RNA was subjected to DNase treatment and cDNA was generated using MMLV reverse transcriptase and oligo $\mathrm{dT}_{18}$. Genespecific primers for qPCR were designed using NCBI primer designer to $X$. laevis transcript reference sequences or as described on Xenbase (Table 1). Melting temperature (Tm) of primers was between 63.6 $69.5^{\circ} \mathrm{C}$. Where possible, primers validated previously were used. A serial dilution of cDNA was used to generate a standard curve for each primer pair to determine primer efficiency. Sample cDNA was diluted to fall within the range of this standard curve. QPCR was performed on a Quantstudio 5 (Applied Biosystems) platform using $2 \times$ SYBR green master mix (Takara Premix Ex Taq). Briefly, a mastermix was made, containing $0.5 \mathrm{uM}$ of each of the corresponding forward and reverse primers and $5 \mathrm{ul}$ of $2 \mathrm{x}$ SYBR green mastermix (Takara). $9 \mu \mathrm{l}$ of this mastermix was added to $1 \mu \mathrm{l}$ of diluted cDNA in a 96-well qPCR plate. Cycling conditions were: $95^{\circ} \mathrm{C} 2 \mathrm{~min}$ followed by 40 cycles at $95^{\circ} \mathrm{C}, 5 \mathrm{~s}$, $62^{\circ} \mathrm{C}, 10 \mathrm{~s}, 72^{\circ} \mathrm{C} 5 \mathrm{~s}$. Reactions were set up in duplicate for each cDNA and target gene and all samples were normalized to the geometric mean of Gapdh and $E f 1 \alpha$ within the same sample to correct for efficiency of reverse transcription. Note that, for oocytes, only Gapdh was used for normalization. The expression levels of genes of interest were calculated using Quantstudio Design and Analysis software (Applied Biosystems), using the standard curve method to normalize the amplification efficiency of target and reference genes as well as run-to-run differences. Specificity of the qPCR was confirmed by the melting curve of amplified products. Each qPCR experiment included a standard curve of each primer set to determine primer efficiency. Primer efficiency lay between $90.7-109 \%$. This efficiency was used in the normalization and fold change calculation.

\section{Data analysis}

All graphs were produced and statistical analyses performed as described using Graph Pad Prism 7.0 software.

\section{Results}

\section{The grem 1 transgene $G$ does not significantly alter fecundity of female Xenopus}

The number of eggs laid in one hour was calculated for each female in the study. Due to the strong correlation between female bodyweight before induction and the number of eggs laid in 1 hour (Table 2, Figure 2a), the data were normalized for pre-induction bodyweight. While $G$ female founders typically laid fewer eggs per hour than either G8 F1

Table 1: Primer sequences

\begin{tabular}{|c|c|c|c|}
\hline Gene & Forward primer sequence (5' - 3') & Reverse primer sequence (5' - 3') & Reference \\
\hline grem 1 & CCTTCCCACAGGATGAACTG & GGCTCCCTGTGATCCACTAA & $114 \mathrm{bp} /(20)$ \\
\hline hsp 70 & CTCATCAAGCGCAACACAAC & CTCCСТCAAACACCTGGATAAG & $100 \mathrm{bp} / \#$ \\
\hline bmp15 & TCATCGGCAGGTGCAAGATT & ACGTCCATCTTGTGTGCCAT & $121 \mathrm{bp}$, this study \\
\hline gapdh & GACATCAAGGCCGCCATTAAGACT & AGATGGAGGAGTGAGTGTCACCAT & $115 \mathrm{bp} / \#$ \\
\hline efla & GCCTTCCCAGGGTTCAACAT & TCGGTGGGTCACTCTTTGAAT & $100 \mathrm{bp} / \#$ \\
\hline
\end{tabular}

\# = Rochester.edu: Jacques Robert Xenopus laevis research resource for immunobiology 
Table 2. Rate of egg laying in G founder, G8 F1 and wild type females

\begin{tabular}{|c|c|c|c|c|}
\hline Female & $\begin{array}{l}\text { Pre-induction } \\
\text { weight (g) }\end{array}$ & $\begin{array}{l}\text { Eggs laid / } \\
\text { hour }\end{array}$ & $\begin{array}{c}\text { Eggs/hour } \\
\text { normalized } \\
\text { for bodyweight }\end{array}$ & $\begin{array}{c}\text { Mean (+/- SD) } \\
\text { normalized }\end{array}$ \\
\hline G3 & 67 & 381 & 353 & \multirow{3}{*}{$307(+/-48)$} \\
\hline G9 & 39 & 162 & 258 & \\
\hline G12 & 62 & 312 & 312 & \\
\hline G8.4 & 49 & 433 & 548 & \multirow{4}{*}{$382(+/-140)$} \\
\hline G8.5 & 62 & 305 & 305 & \\
\hline G8.6 & 51 & 363 & 441 & \\
\hline G8.7 & 77 & 291 & 234 & \\
\hline WT1 & 50 & 250 & 310 & \multirow{7}{*}{$395(+/-120)$} \\
\hline WT2 & 67 & 292 & 270 & \\
\hline WT3 & 63 & 259 & 255 & \\
\hline WT4 & 92 & 852 & 574 & \\
\hline WT5 & 67 & 526 & 487 & \\
\hline WT6 & 55 & 376 & 424 & \\
\hline WT7 & 69 & 499 & 448 & \\
\hline
\end{tabular}

or wild type females (Figure $2 \mathrm{~b}$ ), a one-way ANOVA test showed no significant differences between means $(F=0.69 ; p=0.524)$ of founder $G$ females, G8 F1 females, and wild type animals when egg laying rate was normalized for bodyweight.

\section{Neither maternal nor paternal grem 1 transgene $G$ alters fertility of Xenopus eggs}

We sacrificed five transgenic founder males confirmed to be carrying the $G$ transgene and carried out in vitro fertilization of eggs from a single female WT frog, as well as to different WT females (Figure $1 \mathrm{~b}$ ). The rate of fertilization was calculated as a percentage of eggs reaching 2 to 4 cell stage, for three replicate fertilizations of around 100 eggs each. For male founders back-crossed to the same individual WT female, fertility ranged from $66 \%$ (G6 male) to 93\% (G8 male), with a wild type control male giving $87 \%$ with the same female. A oneway ANOVA showed no significant difference in mean fertilization rate amongst males $(\mathrm{F}=2.53$; $\mathrm{p}=0.09$; Figure $2 \mathrm{c})$.

Similarly, when eggs from female founders $(n=3)$, female F1 derived from G8, and wild type females were fertilized with sperm from the same WT male (Figure 1c), one way ANOVA again showed no significant difference in mean fertilization rate, $(\mathrm{F}=2.58, \mathrm{p}=0.07$, Figure 2d).

\section{Embryos with a grem 1 mother are less likely to survive early embryonic development}

Having found no differences in fecundity or fertility to explain the difficulty breeding $G$ female frogs, we next examined early development. Xenopus embryos are dependent on maternal transcripts until the mid-blastula transition at stage 8 , so we would expect any maternal effect to impact early on in the animal's development. We analysed the percentage of embryos that had been successfully fertilized from each replicate as either normal, dead or poorly gastrulated at stage 14. Survival rates (normal developing percentage) of embryos derived from $G$ females ranges from $3 \%$ to $59 \%$, with wild types at 73 to $92 \%$. Generally, mean survivability was lowest in offspring of females carrying $G$, but there were also differences depending on the founder, indicating that transgene position and context may be important.

One-way ANOVA showed significant variation $(\mathrm{F}=5.35, \mathrm{p}=0.004)$ between treatments with Tukey post-hoc testing indicating significant differences between survival rates of G3 and G9 offspring compared to WT-derived offspring with the same father (Figure 3a). Offspring of G12 and the G8 F1 group both had lower survival than wild types with the same father, although these were not statistically significant. However, if individual G8 lines were compared, G8.4 had significantly lower survival than wild types (data not shown). The better survival in G8.5, 8.6 and 8.7 could be due to mosaicism in these females as they did not produce the expected number of transgenic offspring (see Table 3).This suggests that the degree of effect of a maternal $G$ transgene on embryo early survival depends on transgene context.

Offspring of male frogs carrying $G$ had much better survival to stage 14. Unpaired t-tests were used to compare survival rate for each male's offspring to that of a wild type male crossed with the same female. No significant differences were found between pairs (Figure $3 \mathrm{~b}$ ), indicating no adverse effect on early embryogenesis when the father carries a $G$ transgene. Early survival from each male's offspring when crossed to the same wild type female (Figure $3 \mathrm{c}$ ) also showed no significant variance between crosses (One-way ANOVA, $\mathrm{F}=1.05, \mathrm{p}=0.44$ ). In conclusion, paternal $G$ did not affect survivability up to stage 14 .

\section{Transgenic ratios are not skewed in $G$ offspring}

When the surviving embryos were 6 days old we were able to score them as transgenic or non-transgenic due to the presence or absence of red fluorescent protein RFP in the lenses of the eyes. Chi-squared analysis was used to test for any deviation from expected Mendelian ratios, assuming a single transgene insertion site (i.e. founders are heterozygous at a single locus) (Table 3 , significant $p$ values indicate

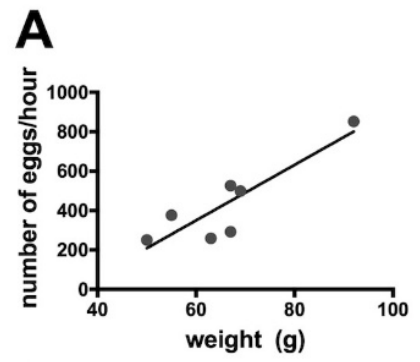

B
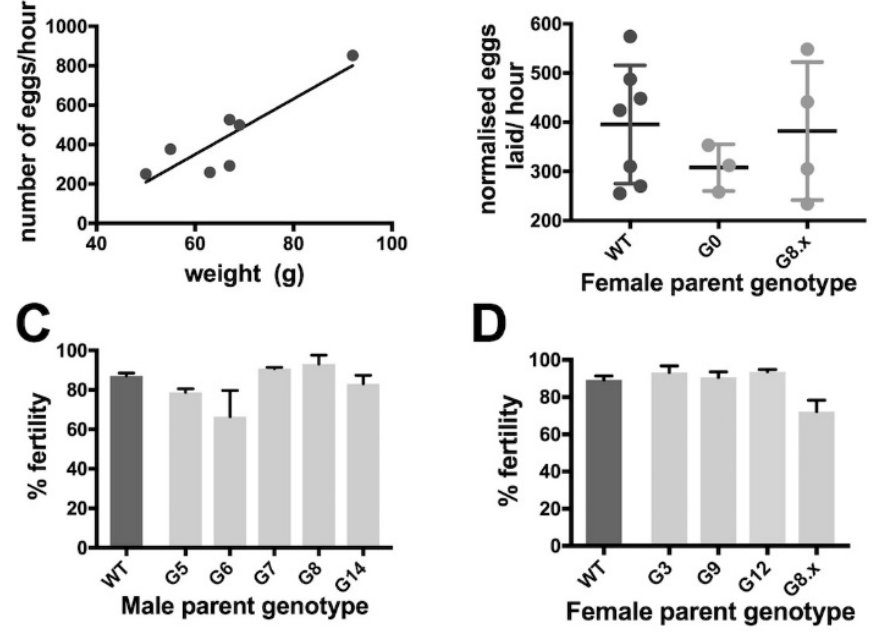

D

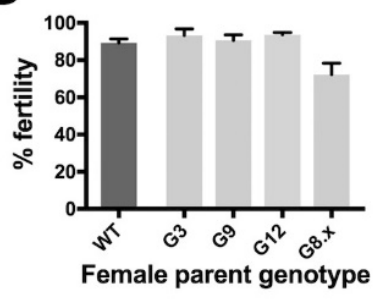

Figure 2. There is no effect of parental transgenic genotype on fecundity or fertilisation rate A) linear regression analysis of seven WT females in the study showing positive correlation between egg laying rate and bodyweight on induction. B) Scatter plot comparing fecundity of three female $\mathrm{G}$ founders (G3, G9, G12), four female G8 F1 and seven wild type female Xenopus. Centre lines show the mean egg laying rate (per hour, normalized for body weight), and error bars are SD. No significant differences were seen between the three groups. C) Column graph showing that mean percentage successful fertilisation rate was not altered when the male parent carried the $\mathrm{G}$ transgene. Sperm from five different founder $\mathrm{G}$ males and one WT male was used to fertilize 3 batches of around 100 eggs from the same WT female and fertilisation scored as the percentage of eggs undergoing successful progression to 4 cells. Error bars are SEM. One-way ANOVA revealed no differences in fertility between any of the male parent genotypes. D) Column graph showing mean percentage fertilisation was not affected when the female parent carried the $\mathrm{G}$ transgene. Sperm from the same WT male was used to fertilise 3 batches of around 100 eggs from each of two WT, three G founders and 4 G F1 females derived from the G8 male. One-way ANOVA revealed no differences in fertility between any of the female parent genotypes 
Table 3. Transgene transmission tracking to offspring of female or male $\mathrm{G}$ heterozygote frogs

\begin{tabular}{|c|c|c|c|c|c|}
\hline \multirow{2}{*}{$\begin{array}{l}\text { Frog with } \\
\text { transgene }\end{array}$} & \multicolumn{3}{|c|}{$\%$} & \multirow{2}{*}{$\begin{array}{c}\text { Chi- } \\
\text { Squared }\end{array}$} & \multirow{2}{*}{$p$-value } \\
\hline & Dead & Wild type & Transgenic & & \\
\hline \multirow{3}{*}{ G3 female } & 68 & 15 & 17 & \multirow{3}{*}{0.11} & \multirow{3}{*}{0.74} \\
\hline & 53 & 26 & 22 & & \\
\hline & 84 & 9 & 8 & & \\
\hline \multirow{3}{*}{ G12 female } & 86 & 6 & 7 & \multirow{3}{*}{0.03} & \multirow{3}{*}{0.85} \\
\hline & 91 & 6 & 3 & & \\
\hline & 89 & 5 & 6 & & \\
\hline \multirow{3}{*}{ G8.4 female } & 65 & 21 & 14 & \multirow{3}{*}{3.86} & \multirow{3}{*}{0.05} \\
\hline & 46 & 39 & 16 & & \\
\hline & 61 & 19 & 19 & & \\
\hline \multirow{3}{*}{ G8.5 female } & 2 & 74 & 23 & \multirow{3}{*}{88.6} & \multirow{3}{*}{$<0.0001$} \\
\hline & 0 & 69 & 31 & & \\
\hline & 0 & 94 & 6 & & \\
\hline \multirow{3}{*}{ G8.6 female } & 16 & 65 & 19 & \multirow{3}{*}{42.12} & \multirow{3}{*}{$<0.0001$} \\
\hline & 96 & 3 & 1 & & \\
\hline & 12 & 69 & 19 & & \\
\hline \multirow{3}{*}{ G8.7 female } & 18 & 58 & 24 & \multirow{3}{*}{6.91} & \multirow{3}{*}{$<0.01$} \\
\hline & 45 & 35 & 19 & & \\
\hline & 30 & 39 & 30 & & \\
\hline & 2 & 49 & 49 & \multirow{3}{*}{1.00} & \multirow{3}{*}{0.31} \\
\hline \multirow[t]{3}{*}{ G5 male } & 0 & 45 & 55 & & \\
\hline & 13 & 35 & 52 & & \\
\hline & 16 & 38 & 46 & \multirow{3}{*}{0.11} & \multirow{3}{*}{0.74} \\
\hline \multirow[t]{3}{*}{ G6 male } & 18 & 42 & 40 & & \\
\hline & 4 & 47 & 49 & & \\
\hline & 11 & 50 & 39 & & \\
\hline \multirow[t]{3}{*}{ G7 male } & 6 & 62 & 32 & 1.94 & 0.16 \\
\hline & 9 & 43 & 48 & & \\
\hline & 18 & 44 & 38 & & \\
\hline G8 male & 15 & 48 & 37 & 1.60 & 0.21 \\
\hline & 6 & 50 & 44 & & \\
\hline & 26 & 43 & 30 & & \\
\hline G14 male & 46 & 34 & 20 & 5.05 & 0.02 \\
\hline & 24 & 45 & 31 & & \\
\hline
\end{tabular}

Deviations from expected Mendelian ratio of 50:50 generate a significant $\mathrm{p}$ value (bold) (Chi-squared test).

deviation). The survival rate for G9 offspring was too low to be meaningful for testing, but appeared Mendelian. G3 and G12 founder females, as well as the G8.4 female, showed the expected ratio (50:50) of transgenic offspring. G8.5, G8.6 and 8.7 generated a higher than expected number of wild types suggesting that these three females might be mosaic for the transgene. All female G8.x are derived from the G8 male, which showed typical Mendelian ratios of transgenic offspring (45\% transgenic). None of the male frogs except G14 yielded nonMendelian ratios of transgenic offspring (39\% transgenic). The balance of transgenic and non-transgenic surviving embryos indicates that maternal, not zygotic, transgene status is responsible for the reduced survival of embryos from $G$ mothers.

\section{Developmental progression up to 10 weeks is not affected by the presence of $G$ transgene or by parent-of-origin}

At two weeks, surviving offspring were transferred to a recirculating aquarium at $22^{\circ} \mathrm{C}$ for 8 weeks, to determine the effect, if any, of tadpole transgene presence, absence or source (i.e. maternal or paternal inheritance). Wild type tadpoles were derived from G3, G9, G12 females and G5 and G7 males; G female founder group were transgenic animals from G3, G9 and G12 mothers; G8.x founder group were transgenic animals from G8.4, 8.5, 8.6, 8.7 mothers, and male founders were transgenic animals from G5, G6, G7, G8 and G14 fathers. There was no difference in median developmental stage at 10 weeks of development: in each group, the median stage was 54 (Figure 3d). One-way ANOVA confirmed that there was no significant variation between groups. Hence, neither the origin or the presence/absence of a $G$ transgene affected developmental stage when tadpoles were reared in normal aquarium temperatures $\left(22^{\circ} \mathrm{C}\right)$.

\section{Ovarian recovery is not affected by the Grem 1 transgene}

Induction of Xenopus female adults with human chorionic gonadotrophin (hCG) normally results in the maturation and laying of all stage VI oocytes. All female Grem $1(\mathrm{G})$ frogs in the study were sacrificed at 6 weeks after induction and the ovaries extracted. This time is expected to allow for full regeneration, replenishing stage VI oocytes [13]. A single lobe from each ovary was isolated for each female and treated with collagenase to separate the oocytes. Oocytes were than staged and counted according to Dumont as stage II to VI, or atretic (dying). Some stage I oocytes were also observed but as many were lost on collagenase treatment, they could not be reliably counted [13].

Interestingly, frogs were weighed before sacrifice and found to have lost around $25 \%$ of their bodyweight compared to pre-induction. Thus,
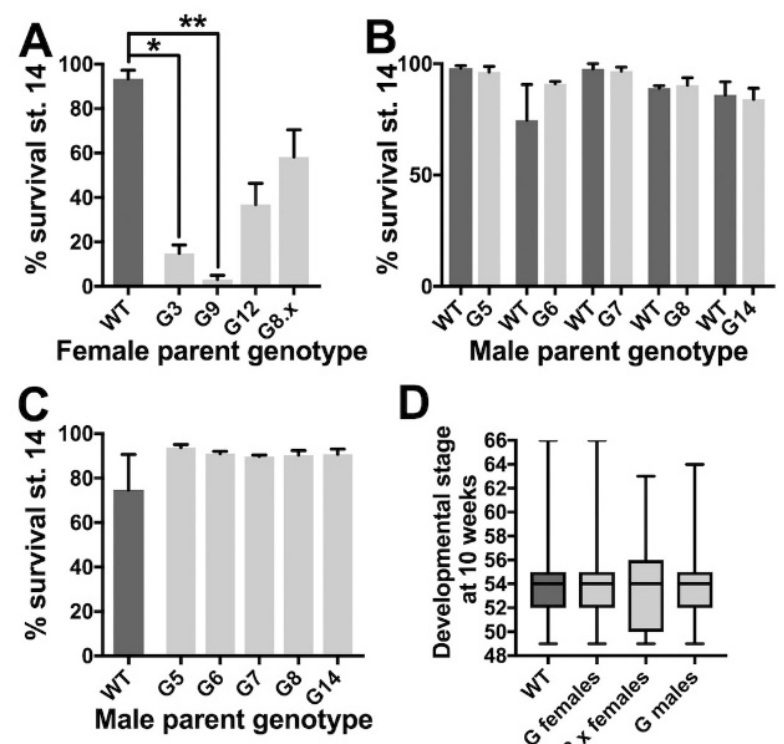

D

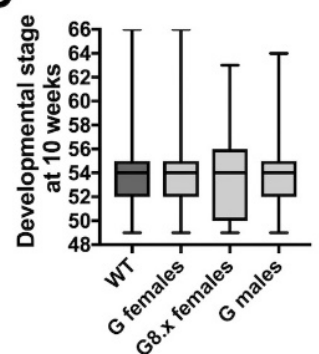

WT vs. transgenic offspring developmental stage at 10 weeks

Figure 3. The low number of offspring from $\mathrm{G}$ females results from poor survival in very early embryonic development

A-C Column graphs showing the $\%$ of fertilised embryos that survive to stage 14 (the end of gastrulation). Columns represent the means of 3 replicate fertilisations of around 100 embryos each, and error bars are SEM. A) Offspring of females carrying a $G$ transgene do not survive as well as those from WT female even when the father is the same. One-way ANOVA with Tukey post hoc pairwise testing showed G3 $\left(\mathrm{p}<0.05,{ }^{*}\right)$ and $\mathrm{G} 9\left(\mathrm{p}<0.01,{ }^{* *}\right)$ have significantly lower survival compared to wild types. B) No differences were observed between early stage survivability in offspring of five different male $G$ founder frogs, when compared to the offspring of WT males with the same mother. C) Similarly, when all five $G$ males were crosses to the same wild type female, early development was unaffected. D) Box plot of developmental stage at 10 weeks post fertilisation for offspring grouped by genotype. Median developmental stage is depicted by the line, with boxes representing first and third quartile, and whiskers showing the minimum and maximum stage, up to stage 66 which is a fully metamorphosed juvenile frog. Median stage at 10 weeks was unaffected by the $G$ transgene regardless of parental or individual genotype. Genotype sample sizes were: WT $\mathrm{n}=145, G$ transgenics derived from carrier females $\mathrm{n}=132, G$ transgenics derived from G8.x females $\mathrm{n}=47$ and $G$ transgenics derived from $G$ males $\mathrm{n}=99$ 

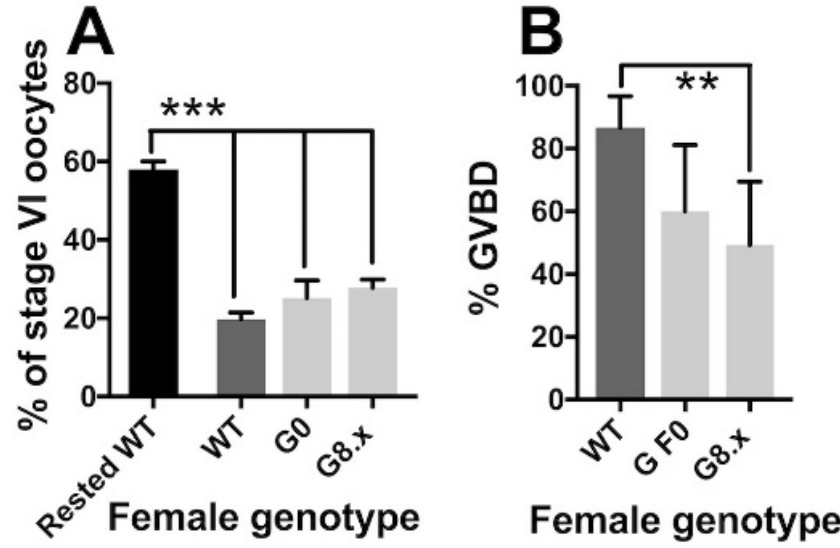

Figure 4. Ovarian recovery and maturation of stage VI oocytes is normal in G female frogs Column graphs showing mean and error bars showing SEM. Grouped transgenic sample sets as for previous figures. A) The percentage of stage VI oocytes in a randomly chosen left and right ovary lobe from WT $(n=2), G$ founders $(n=3)$ or G8 F1 $(n=4)$ generation female frogs, representing the amount of ovarian recovery. The presence of the maternal transgene did not significantly the percentage of stage VI oocytes when compared with wild types that had been induced at the same time (6 weeks prior). However, none of the ovaries in the study had fully regenerated 6 weeks after induction. "Rested WT" shows comparative data from a WT female last induced at $>1$ year prior to sacrifice, and all 3 groups had significantly fewer stage VI oocytes than this female (1 way ANOVA with post hoc Tukey test, $\mathrm{p}<0.001 * * *$ ). B) Percentage of stage VI oocytes responding appropriately to progesterone treatment, scored by the presence of the germinal vesicle as a white spot at the animal pole of the oocyte, and indicating successful maturation in vitro. Fisher's exact test showed significantly fewer G8.x oocytes responded to progesterone compared to WT $(\mathrm{p}<0.01 * *)$

even after 6 weeks, the ovary is not fully recovered as suggested by Dumont. This was also reflected in the percentage of stage VI oocytes, shown in Figure 4a. While a female wild type that had not been induced for 1 year had $58 \%$ oocytes at stage VI, induced wild types had 19 and $20 \%$ respectively, with G founders ranging from 16 to $36 \%$ and G8 F1 females $29-32 \%$. Data were grouped into G founders (F0), G8 F1 and $\mathrm{WT}$, and analysed by one way ANOVA, no significant variance was detected $(\mathrm{F}=1.42, \mathrm{p}=0.27)$. However, if the wild type female that had not been induced 6 weeks previously was included, a significant difference could be detected between this animal and the other study groups (Figure 4a). This suggested that ovarian regeneration was incomplete, but similar, in transgenic and non-transgenic females, provided the regeneration time was consistent. Histological and ultrastructural analysis of wild type vs transgenic ovary samples showed no obvious phenotypic differences in the ovary (data not shown).

\section{Response of stage VI oocytes to progesterone is unaffected by the presence of a $\mathrm{G}$ transgene}

Stage VI oocytes are arrested in G2/M meiotic prophase I, and cannot be fertilized. Exposure to progesterone initiates progression to metaphase II and germinal vesicle breakdown (the breakdown of the egg nuclear membrane, termed GVBD). Oocytes that have undergone GVBD have a white spot at the animal pole, resulting from displacement of pigment from the egg cortex by the nucleus. Some of the stage VI oocytes from $\mathrm{G}$ females appeared less responsive to progesterone (Figure $4 \mathrm{~b}$ ), and Fisher's exact test showed G8.x oocytes to be significantly less likely to mature on progesterone exposure $(\mathrm{p}=0.0035)$. Grouping all $\mathrm{G}$ female oocytes and comparing to WT also revealed a significant reduction in numbers of matured oocytes (Fisher's exact test, $\mathrm{p}=0.0137$ ).
Q-RT-PCR reveals a reduced level of grem 1 transcripts in the ovary of G founders and G8 F1 compared to that of nontransgenics

Initially, we thought that grem1 might be transiently activated from the hsp70:grem 1, $\gamma$-crystallin:RFP (G) transgene during oogenesis, leading to poor development of offspring from $G$ transgene carrying mothers. Previous work has shown that endogenous $h s p 70$ is heat inducible, but normally silent in stage VI oocytes and constitutively active in younger, vitellogenic oocytes [21]. This suggests that the heat shock factors that bind to and activate the endogenous $h s p 70$ locus could also be driving expression from the transgene. To determine if grem 1 was being expressed from the $h s p 70$ transgenic promoter, we used qRT-PCR of total RNA extracted from wild type and transgenic oocytes and ovaries, harvested 6 weeks after initial induction of egg laying. As primers cannot not distinguish between endogenous grem 1 and transgene grem 1 transcripts, this assay measures both together. Unexpectedly, we saw a consistently lower grem1 expression in stage I to VI oocytes from $G$ females when compared to their wild type counterparts (Figure 5a). Significant reduction in grem 1 transcripts was seen at stage $\mathrm{V}$ and VI (Bonferroni multiple comparisons test for WT and $\mathrm{G}$ at each oocyte stage).

To further investigate this surprising result, we analysed grem 1 expression in both ovaries of every frog in the study. Collectively, both the G0 founders and the G8.x F1 female ovaries expressed significantly lower grem 1 than wild types (Figure $5 c, 1$ way ANOVA, with Tukey post-hoc testing $\mathrm{p}<0.0001)$. This was due to more than 2 -fold down regulation of grem 1 in all the lines (Figure 5b), with G9 the most affected at almost 5-fold down regulated. Interestingly, G9 showed the poorest survival rate (refer to Figure 2a), with just 3\% of embryos surviving past gastrulation.

Surprisingly then, the presence of the transgene in the mother results in a decrease in grem 1 transcripts. This suggests that the endogenous grem 1 locus is less active in the presence of the $\mathrm{G}$ transgene. To see if endogenous $h s p 70$ was influenced by the transgene, we also measured $h s p 70$ transcripts but found no difference in expression between wild type and transgenic ovaries (Figure 5d, 1 way ANOVA, Tukey posthoc testing). Since activation of the transgene should also result in activation of the endogenous locus, increasing $h s p 70$ transcripts, this suggests that the transgene is not active, or leaky, in the ovary, and that there is no significant effect of transgene presence on the endogenous hsp70 locus, at least in this tissue.

To look at another locus for comparison, we measured bmp15 transcripts, as BMP15 levels in follicular fluid are a well-documented indicator of oocyte quality and successful early development in humans. bmp15 expression varied between the three groups (Figure 5e, 1 way ANOVA $\mathrm{F}=6.33, \mathrm{p}=0.003$ ) and was significantly higher in G8.x compared to either G0 founders or WT ovaries, (Tukey post hoctesting, $\mathrm{p}=0.009$ and 0.010 respectively). However we did not observe a strong correlation between $b m p 15$ and grem 1 transcript levels across all samples, indicating that the reduced survival of $\mathrm{G}$ female offspring is not due to altered bmp15 expression.

\section{Discussion}

\section{Grem 1 may be a pan-vertebrate marker of oocyte competence}

In humans, the success of assisted fertility depends on being able to predict oocyte viability (Reviewed by [22]). The competence of the oocyte to mature, fertilize, develop and implant into the uterine wall 
A

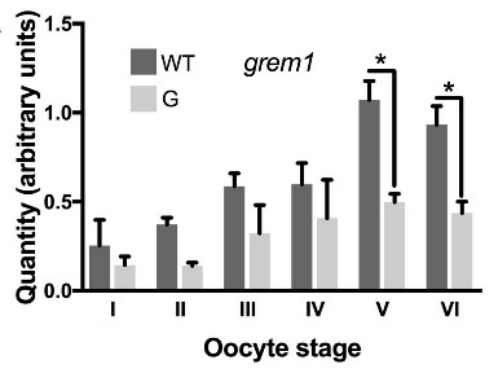

C

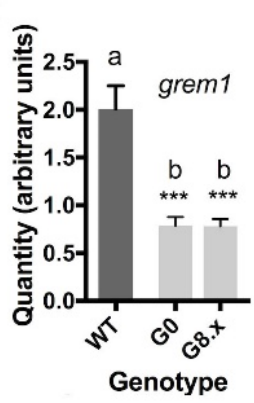

$B$

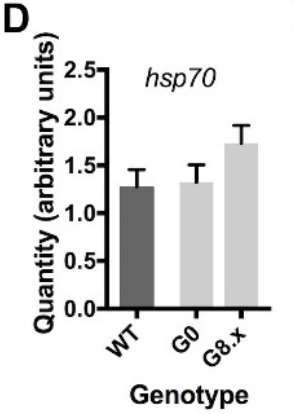

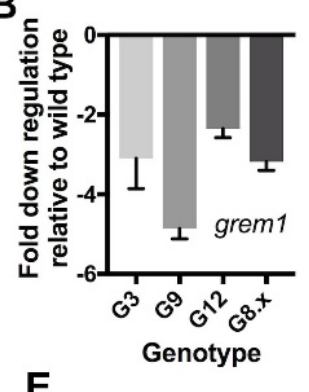

$\mathbf{E}$

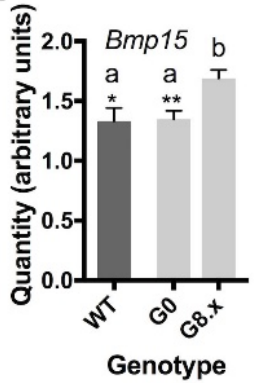

Figure 5. Q-RT-PCR revealed a decrease in whole ovary grem1 that correlates with poor survivability of the offspring of $G$ mothers

A-E) Column graphs of transcript levels measured by q-RT-PCR for oocytes (A) or a randomly chosen left and right ovary lobe (B-E) from each animal. Samples were tested in triplicate and normalized to the geometric mean of two housekeepers, Gapdh and EFlalpha, except in A where normalization was to Gapdh. In B, columns show means of relative quantity grem 1 transcripts in $\mathrm{G}$ ovaries relative to wild type. In C-E, columns depict means of technical and biological replicates for each group of genotypes and with error bars are SEM. Quantities are expressed as arbitrary values calculated from standard curves. A) Grem1 is expressed at lower levels in oocytes from $\mathrm{G}$ females at all stages of oocyte development, this is significant for stage V and VI oocytes. Data is the mean of triplicate data from two G females, G8.4 and G3, and two wild type (WT) females. B) Grem 1 transcripts are at least 2 fold lower in G ovaries than in wild type, with G9 (which had the poorest survival) having nearly 5 -fold reduction in grem 1 transcripts. C) Grem1 is expressed at significantly lower levels than WT in either G founders or G8.x ovaries. C) $H s p 70$ transcripts are not significantly different between WT and G ovaries. D) BMP15 transcript levels were significantly higher in the G8 F1 ovaries, than either WT or G founders. ${ }^{*} \mathrm{p}<0.05, * * \mathrm{p}<0.01,{ }^{* * *} \mathrm{p}<0.001$

will determine the rate of successful pregnancy. It is highly desirable to be able to identify the most competent oocytes to avoid having to transfer multiple embryos and risk multiple pregnancies. Oocyte competence is known to be closely associated with normal follicular development, with intrinsic reciprocal signals between the oocyte and its surrounding follicle cells playing as key role, as well as extrinsic, circulating hormonal factors. Here, we have shown a correlation between maternal grem 1 and early embryonic survival in the highly fecund frog Xenopus laevis. Although we cannot rule out an effect of the presence of the transgene in mothers on extrinsic hormone regulation, our results favour an intrinsic effect on ovarian follicle cells. The presence of the $\mathrm{G}$ transgene in follicle cells results in significantly lower levels of grem 1 transcripts in the ovary, suggesting that the transgene somehow results in repression of the endogenous grem 1 gene. Lower levels of grem 1 transcripts would be expected to reduce available Grem1 protein, potentially resulting in de-repression of maternal BMP4/7 signalling. While the exact mechanism remains unknown, candidate gene approaches have shown that grem 1 transcript abundance is also a predictor of human oocyte competence $[17,18]$. In an unbiased microarray study, Assidi et al also identified grem 1 as one of a handful of markers associated with oocyte competence in a bovine model [23]. Taken together with our results in the frog model, Grem1 levels seem

to be a marker of oocyte competence in highly fecund non-mammalian as well as mammalian vertebrates. An exception to this is the mouse, where conditional, ovary-specific grem 1 null mothers showed normal fertility [24]. A possible explanation for this is that grem2, which is also expressed in the follicle cells, may compensate for loss of grem 1 in mouse ovaries. Grem2, which is not found in Xenopus, seems have a conserved role in regulation of primordial follicle transition in some mammals $[25,26]$. However, in mice, grem 1 has been shown to play this role [24]. Some flexibility in the roles of the two orthologues therefore exists in mammals.

\section{Maternal presence of the $G$ transgene in Xenopus results in poor survival in early embryogenesis}

We had previously observed that it was difficult to obtain viable offspring from $G$ female founders, which carry randomly inserted hsp70:grem 1, $\gamma$ crys:RFP transgene at a single locus. We have confirmed reduced viability is independent of the transgenic status of the oocyte or the location of the transgene, and shown that oogenesis, oocyte maturation, fecundity and fertilization of eggs from $\mathrm{G}$ females does not differ from that of wild type frogs. The reduction in viable offspring was found to result from reduced survival in early development of the embryos with $\mathrm{G}$ mothers, regardless of the embryo's own transgenic status. Survival of offspring of $\mathrm{G}$ mothers from fertilization to the end of gastrulation is significantly lower than that of embryos with wild type parents or a $\mathrm{G}$ father. At $18^{\circ} \mathrm{C}$, transgenic and wild type offspring survive at expected Mendelian ratios, suggesting no adverse effect of the embryo's own genotype. Embryos from $G$ mothers that survive early embryogenesis go on to develop at the same rate and with the same success as their wild type half-siblings, or embryos with $\mathrm{G}$ fathers. The presence of a maternal $G$ transgene is therefore most likely to be reducing embryo viability due to its presence in the follicle cells of the ovary.

\section{Reduced grem 1 expression in the Xenopus ovary may result from transgene suppression}

The most likely explanation for the reduced Grem 1 in ovaries of G female frogs is that the presence of the transgene is somehow supressing the transcription of the endogenous grem 1 locus. This effect was seen to varying degrees in different lines, suggesting that integration position has some effect on the amount of repression. grem 1 transcripts were consistently reduced, relative to wild type controls, at all stages of oocyte development (data not shown) and in all 4 lines tested. Transgene silencing has been studied extensively in plants, where its discovery helped elucidate mechanisms of epigenetic silencing such as RNAi. In plants, two types of silencing are known (reviewed in [27]). Position effects, where the transgene is negatively regulated by flanking DNA or location in heterochromatic regions, seem unlikely to be the cause of the silencing we observe here, since each line would be expected to have the transgene integrated in a different random location. Furthermore, the $h s p 70$ :grem 1 transgene is linked to $\gamma C r y s: R F P$, allowing identification of transgenic animals without genotyping, and clearly this is not silenced. The second mechanism is termed homology-dependent gene silencing (HDGS) which may result from multiple transgenes integrating at one site in random orientation, which may result in inverted repeats that can interact with, and silence, homologous sequences in the genome. HDGS seems the more likely explanation, since it could explain why grem 1 transcripts, but not $h s p 70$ transcripts, are significantly reduced. Similar transgene silencing observed in mammals has been shown to arise from inverted repeats of a transgene [28] although the mechanism was not able to be fully characterised. 


\section{Conclusions}

Female frogs carrying a heat-inducible transgene driving conditional expression of grem 1 produce few viable offspring. Here we have shown that this is not due to reduced fecundity or fertilization rate, but that the offspring of these females die in early embryogenesis regardless of whether or not they themselves carry the transgene. This maternal effect lethality is not caused by transgene leakage, which would elevate grem 1 expression, as we observe reduced levels of grem 1 transcripts in the Xenopus ovary of transgenic mothers. Therefore, it is likely Grem 1 levels are associated with chance of survival in the early stages of embryogenesis in frogs as they have been found to in mammals $[17,18,22]$. Furthermore, this indicates that transgene suppression of an endogenous locus may occur in the Xenopus female germline, an unexpected finding. The mechanism by which this occurs is presently unknown, but could be a case of homology-dependent gene silencing.

\section{Acknowledgements}

CWB conceived the study, designed the experiments, analysed data and wrote the manuscript. LT and CWB performed all Xenopus breeding and experiments and CB and JW performed all qRT-PCR analysis. All authors read and approved the final manuscript. We are grateful to Matthew Downes for his expertise in electron microscopy studies even though this data did not make the final cut. Mark Lokman kindly read and commented on a draft and provided a second opinion on statistics. Jolyn Chia, Nikita Woodhead and Nat Lim take excellent care of our frog colony, and Valerie Gavois arranged the internships for $\mathrm{CB}$ and LT who are students at Ecole Nationale Vétérinaire de Toulouse, France.

\section{References}

1. Venkatesh T, Suresh PS, Tsutsumi R (2014) New insights into the genetic basis of infertility. Appl Clin Genet 7: 235-243. [Crossref]

2. Dixit H, Rao LK, Padmalatha VV, Kanakavalli M, Deenadayal M, et al. (2006) Missense mutations in the BMP15 gene are associated with ovarian failure. Hum Genet 119: 408-415. [Crossref]

3. Persani L, Rossetti R, Cacciatore C (2010) Genes involved in human premature ovarian failure. J Mol Endocrinol. 45:257-79.

4. Norling A, Hirschberg AL, Rodriguez-Wallberg KA, Iwarsson E, Wedell A, et al. (2014) Identification of a duplication within the GDF9 gene and novel candidate genes for primary ovarian insufficiency (POI) by a customized high-resolution array comparative genomic hybridization platform. Hum reprod. 29:1818-27. [Crossref]

5. Galloway SM, McNatty KP, Cambridge LM, Laitinen MP, Juengel JL, et al. (2000) Mutations in an oocyte-derived growth factor gene (BMP15) cause increased ovulation rate and infertility in a dosage-sensitive manner. Nat genet. 25:279-283. [Crossref]

6. Martinez-Royo A, Jurado JJ, Smulders JP, Marti JI, Alabart JL, et al. (2008) A deletion in the bone morphogenetic protein 15 gene causes sterility and increased prolificacy in Rasa Aragonesa sheep. Anim genet. 39:294-297. [Crossref]

7. Nicol L, Bishop SC, Pong-Wong R, Bendixen C, Holm LE, et al. (2009) Homozygosity for a single base-pair mutation in the oocyte-specific GDF9 gene results in sterility in Thoka sheep. Reproduction 138: 921-933. [Crossref]

8. Juengel JL, Hudson NL, Berg M, Hamel K, Smith P, et al. (2009) Effects of active immunization against growth differentiation factor 9 and/or bone morphogenetic protein 15 on ovarian function in cattle. Reproduction 138: 107-114. [Crossref]
9. Dong J, Albertini DF, Nishimori K, Kumar TR, Lu N, et al. (1996) Growth differentiation factor-9 is required during early ovarian folliculogenesis. Nature 383 : 531-535. [Crossref]

10. Yan C, Wang P, DeMayo J, DeMayo FJ, Elvin JA, Carino C, et al. (2001) Synergistic roles of bone morphogenetic protein 15 and growth differentiation factor 9 in ovarian function. Mol Endocrinol. 15:854-866. [Crossref]

11. Brazil DP, Church RH, Surae S, Godson C, Martin F (2015) BMP signalling: agony and antagony in the family. Trends Cell Biol 25: 249-264. [Crossref]

12. Korchynskyi O, ten Dijke P (2002) Identification and functional characterization of distinct critically important bone morphogenetic protein-specific response elements in the Id1 promoter. J Biol Chem 277: 4883-4891. [Crossref]

13. Dumont JN (1972) Oogenesis in Xenopus laevis (Daudin). I. Stages of oocyte development in laboratory maintained animals. J Morphol 136: 153-179. [Crossref]

14. Brazil DP, Church RH, Surae S, Godson C, Martin F (2015) BMP signalling: agony and antagony in the family. Trends Cell Biol 25: 249-264. [Crossref]

15. Hsu DR, Economides AN, Wang X, Eimon PM, Harland RM (1998) The Xenopus dorsalizing factor Gremlin identifies a novel family of secreted proteins that antagonize BMP activities. Mol Cell. 1:673-83.

16. Wang YH, Keenan SR, Lynn J, McEwan JC, Beck CW (2015) Gremlin1 induces anterior-posterior limb bifurcations in developing Xenopus limbs but does not enhance limb regeneration. Mech Dev 138 Pt 3: 256-267. [Crossref]

17. McKenzie LJ, Pangas SA, Carson SA, Kovanci E, Cisneros P, et al. (2004) Human cumulus granulosa cell gene expression: a predictor of fertilization and embryo selection in women undergoing IVF. Hum reprod. 19:2869-2874.

18. Cillo F, Brevini TA, Antonini S, Paffoni A, Ragni G, et al. (2007) Association between human oocyte developmental competence and expression levels of some cumulus genes. Reproduction 134: 645-650. [Crossref]

19. Nieuwkoop PD, Faber J. A Normal Table of Xenopus laevis (Daudin). Amsterdam Elsevier/North Holland; 1967.

20. Pearl EJ, Barker D, Day RC, Beck CW (2008) Identification of genes associated with regenerative success of Xenopus laevis hindlimbs. BMC Dev Biol. 8:66.

21. Gordon S, Bharadwaj S, Hnatov A, Ali A, Ovsenek N (1997) Distinct stress-inducible and developmentally regulated heat shock transcription factors in Xenopus oocytes. Dev Biol 181: 47-63. [Crossref]

22. Li Q, McKenzie LJ, Matzuk MM (2008) Revisiting oocyte-somatic cell interactions: in search of novel intrafollicular predictors and regulators of oocyte developmental competence. Mol Hum Reprod. 14:673-678.

23. Assidi M, Dufort I, Ali A, Hamel M, Algriany O, et al. (2008) Identification of potential markers of oocyte competence expressed in bovine cumulus cells matured with folliclestimulating hormone and/or phorbol myristate acetate in vitro. Biol Reprod 79: 209222. [Crossref]

24. Myers M, Tripurani SK, Middlebrook B, Economides AN, Canalis E, et al. (2011) Loss of gremlin delays primordial follicle assembly but does not affect female fertility in mice. Biol Reprod 85: 1175-1182. [Crossref]

25. Nilsson EE, Larsen G, Skinner MK (2014) Roles of Gremlin 1 and Gremlin 2 in regulating ovarian primordial to primary follicle transition. Reproduction 147: 865874. [Crossref]

26. Ikeda Y, Hasegawa A, Tsubamoto H, Wakimoto Y, Kumamoto K, et al. (2016) Effects of gremlin-2 on the transition of primordial follicles during early folliculogenesis in the human ovary. Eur J Obstet Gynecol Reprod Biol. 203:72-77.

27. Rajeevkumar S, Anunanthini P, Sathishkumar R (2015) Epigenetic silencing in transgenic plants. Front Plant Sci 6: 693. [Crossref]

28. Calero-Nieto FJ, Bert AG, Cockerill PN (2010) Transcription-dependent silencing of inducible convergent transgenes in transgenic mice. Epigenetics Chromatin. 3:3.

Copyright: $\odot 2018$ Beck CW. This is an open-access article distributed under the terms of the Creative Commons Attribution License, which permits unrestricted use, distribution, and reproduction in any medium, provided the original author and source are credited. 